\title{
PHYSIOLOGIC EFFECTS OF EXTRACELLULAR SUPEROXIDE DISMUTASE TRANSGENE OVEREXPRESSION ON MYOCARDIAL FUNCTION AFTER ISCHEMIA AND REPERFUSION INJURY
}

Edward P. Chen, MD

Hartmuth B. Bittner, MD, PhD

R. Duane Davis, MD

Peter Van Trigt, MD

Rodney J. Folz, MD, PhD
Objective: Myocardial injury after ischemia and reperfusion may be mediated, in part, by oxygen-derived free radicals. In this study the protective effects of extracellular superoxide dismutase overexpression were directly assessed in the hearts of transgenic mice, after ischemia and reperfusion injury, using an isolated work-performing murine heart preparation and computerized analysis of functional data. Methods: A blinded study was performed to compare cardiac function in the hearts of both transgenic mice with a 3.5-fold overexpression of myocardial extracellular superoxide dismutase ( $n=6,22$ to $26 \mathrm{gm})$ and littermate controls $(n=8,22$ to $26 \mathrm{gm})$. Preload-dependent cardiac output, contractility, heart rate, stroke work, and stroke volume were evaluated in the two groups before and after a 6-minute period of normothermic ischemia. Results: No differences were found between extracellular superoxide dismutase hearts and control hearts in any parameter of myocardial function before ischemia. After ischemia, decreases in cardiac output occurred in both groups; however, this decrease was larger in control mice compared with extracellular superoxide dismutase mice. A higher percentage of recovery was also observed in the contractility, heart rate, stroke work, and stroke volume of extracellular superoxide dismutase hearts compared with control hearts. Conclusion: After global normothermic ischemia and subsequent reperfusion, decreases in cardiac function occurred in both extracellular superoxide dismutase and control mice; however, a higher percentage of recovery was observed in the extracellular superoxide dismutase overexpressed hearts. These data suggest that extracellular superoxide dismutase transgene overexpression significantly improves preservation of myocardial function after ischemia and reperfusion injury. (J Thorac Cardiovasc Surg 1998;115:450-9)
$\mathrm{M}$ yocardial injury after ischemia and reperfusion may be mediated by oxygen-derived free radicals and is supported by the observation that signif-

From the Division of Cardiovascular and Thoracic Surgery and the Division of Pulmonary and Critical Care Medicine (R.J.F.), Duke University Medical Center, Durham, N.C.

Dr. Chen is a recipient of a National Research Service Award, Fellowship No. HL09489

Read at the Seventy-seventh Annual Meeting of The American Association for Thoracic Surgery, Washington, D.C., May 4-7, 1997.

Received for publication May 6, 1997; revisions requested May 30, 1997; revisions received Oct. 28, 1997; accepted for publication Oct. 29, 1997.

Address for reprints: Edward P. Chen, MD, University of California, San Francisco, S-343, Box 0470, San Francisco, CA 94143.

Copyright (C) 1998 by Mosby, Inc.

$0022-5223 / 98 \$ 5.00+0 \quad \mathbf{1 2 / 6 / 8 7 2 2 1}$ icant quantities of these radicals are generated during postischemic reperfusion. Such species include the superoxide anion, hydrogen peroxide, the hydroxyl radical, and the peroxynitrite anion. These radicals damage normal tissue through the peroxidation of lipids and oxidation of sulfhydryl groups, which eventually leads to deficiencies in cell membrane permeability and enzyme function. ${ }^{1}$

Several nonenzymatic and enzymatic defense mechanisms occur naturally in aerobic organisms to potentially protect against oxidant-mediated injury. Nonenzymatic antioxidant substances include vitamin $\mathrm{E}$, vitamin $\mathrm{C}$, vitamin $\mathrm{B}$, and $\beta$-carotene. ${ }^{2}$ Enzymatic antioxidants include catalase and glutathione, which both detoxify hydrogen peroxide through separate biochemical pathways. Superoxide dismutase (SOD) represents a third enzymatic antioxidant that has been shown to catalyze the dismu- 


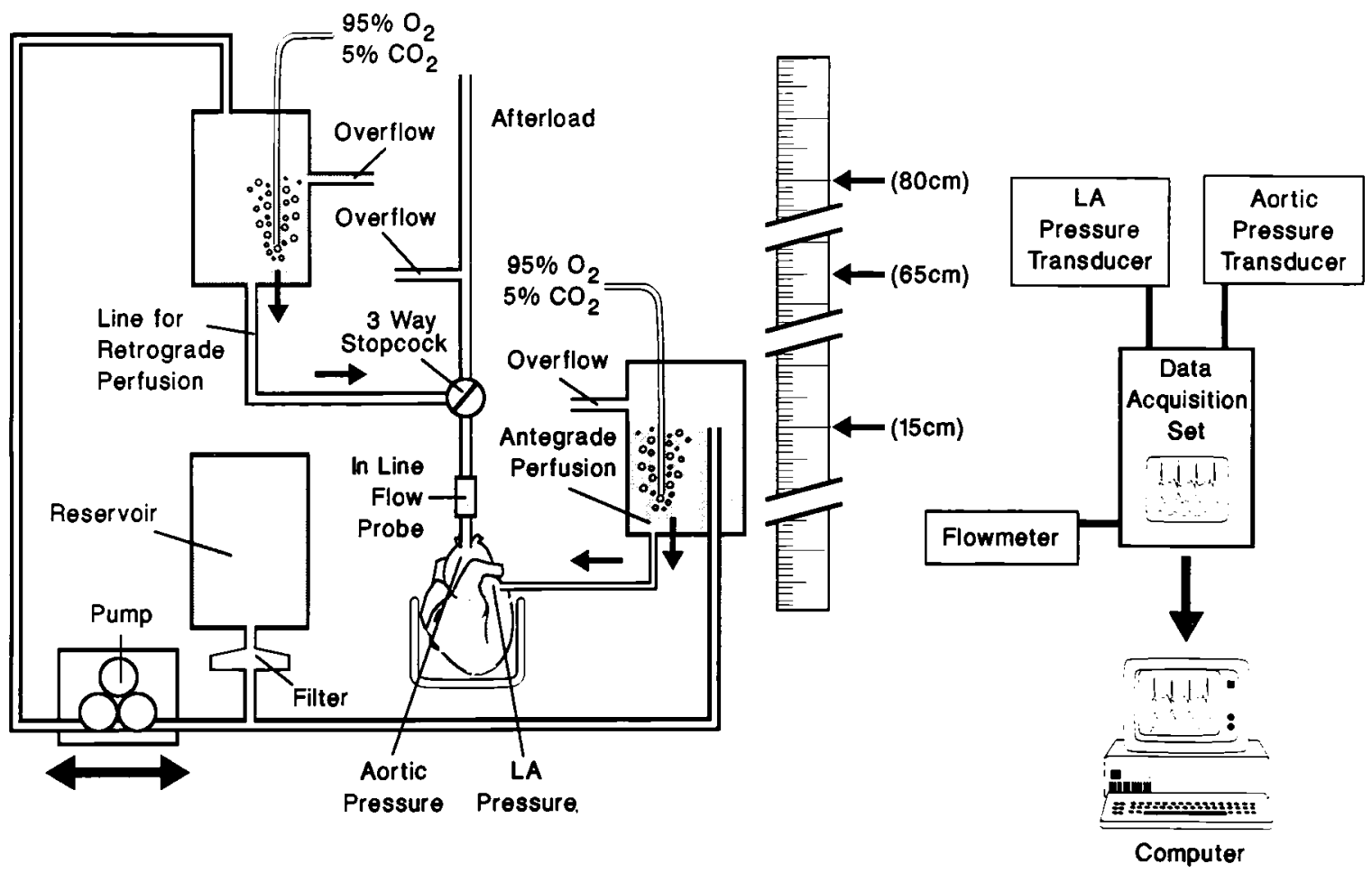

Fig. 1. The isolated work-performing murine heart preparation. The key features of this system include jacketed water baths, reservoirs, and water lines to minimize heat loss; an in-line ultrasonic flow probe to measure cardiac output; aortic and left atrial $(L A)$ micromanometers; and a computerized data acquisition and analysis system.

tation of the superoxide anion to hydrogen peroxide and molecular oxygen. ${ }^{3}$

Controversy remains, however, as to the extent that antioxidants protect the heart after ischemia and reperfusion injury. Recent developments in transgenic technology represent a unique strategy for elucidating any role individual antioxidants may play in preserving myocardial function in this setting. This study was therefore designed to directly assess the myocardial protective effects of extracellular superoxide dismutase (EC-SOD) overexpression in the hearts of transgenic mice after ischemia and reperfusion injury, using an isolated workperforming murine heart preparation ${ }^{4}$ and computerized analysis of functional data.

\section{Materials and methods}

Study design, experimental groups, and generation of transgenic mice. In a blinded study, six transgenic mice with myocardial overexpression of the human EC-SOD (2 to 4 months, 22 to $26 \mathrm{gm}$ ) were evaluated and compared with eight littermate control mice (CTL, 2 to 4 months, 22 to $26 \mathrm{gm})$ in terms of preischemic and postischemic cardiac function.
The transgenic mice used in this investigation had previously been created by Oury and associates ${ }^{5}$ by use of an expression vector comprised of the human $\beta$-actin promoter coupled to a full-length cDNA encoding for the human EC-SOD. This linearized transgene construct was microinjected into the pronuclei of fertilized single-cell mouse embryos, which were then reimplanted into the oviduct of an adult female mouse. Offspring were screened for the presence of the transgene by Southern blot and/or polymerase chain reaction analysis of tail DNA. A founder mouse was bred with $\mathrm{B} 6 \mathrm{C} 3$ (F1) mice. Myocardial expression of human EC-SOD messenger RNA in the transgenic mice used in this report was confirmed using a well-established technique of reverse transcriptase polymerase chain reaction. ${ }^{6}$ Cytochrome $c$ reduction inhibition ${ }^{7}$ was used to measure myocardial EC-SOD activity in both transgenic and nontransgenic hearts. $^{5}$

Isolated work-performing murine heart apparatus. The isolated work-performing (Langendorff) murine heart preparation (Fig. 1) used for acquisition of functional data in this study is previously well described. ${ }^{4}$ An in-line ultrasonic $2 \mathrm{~N}$ flow probe (Transonic System, Inc., Ithaca, N.Y.) was connected to the aortic root cannula to measure aortic flow. Aortic and left atrial pressures were measured with in-line 5F micromanometers (Millar Instruments, Inc., Houston, Tex.). Transition from retro- 
grade perfusion (through the aortic cannula) to antegrade perfusion (through the left atrial cannula) or vice versa was easily done in the same heart by clamping off one system while the other was being used. The isolated heart was immersed in a jacketed, $38^{\circ} \mathrm{C}$ water bath filled with Krebs-Henseleit solution (composed of $118 \mathrm{mmol} / \mathrm{L} \mathrm{NaCl}$, $4.7 \mathrm{mmol} / \mathrm{L} \mathrm{KCl}, 7 \mathrm{mmol} / \mathrm{L} \mathrm{MgSO}_{4}, 1.2 \mathrm{mmol} / \mathrm{L} \mathrm{KH}_{2} \mathrm{PO}_{4}$, $7 \mathrm{mmol} / \mathrm{L} \mathrm{NaHCO}, 11.1 \mathrm{mmol} / \mathrm{L}$ glucose, $3 \mathrm{mmol} / \mathrm{L}$ $\mathrm{CaCl}_{2}$, and $0.5 \mathrm{mmol} / \mathrm{L} \mathrm{NaEDTA}$ ). Krebs-Henseleit solution was also circulated in the apparatus and maintained at an average temperature between $38.0^{\circ} \mathrm{C}$ and $38.5^{\circ} \mathrm{C}$ using an in-line water pump and heating coils. Blindtipped catheters were used to oxygenate the buffering solutions in the reservoirs with $95 \%$ oxygen and $5 \%$ carbon dioxide. Blood gases of the buffered solutions were checked periodically throughout the experiments to ensure physiologic levels of $\mathrm{pH}$, oxygen, carbon dioxide, and electrolytes (Gem-Stat Mallinckrodt Sensor Systems, Ann Arbor, Mich.).

Anesthesia and operation. The anesthetic regimen and operative procedure have been previously well described. ${ }^{4}$ A clam-shell incision was made to expose the mediastinum and pleural cavities, and the thymus was bluntly dissected off the pericardium. The pericardium was opened, and the heart was cold arrested with $4^{\circ} \mathrm{C}$ KrebsHenseleit solution and excised from the chest. The aortic root was then cannulated with a $2 \mathrm{~mm} 20$-gauge angiocatheter at the level of the innominate artery.

Experimental protocol. Retrograde perfusion was immediately begun with warm Krebs-Henseleit solution and maintained at a pressure of $60 \mathrm{~mm} \mathrm{Hg}$ to ensure adequate coronary artery perfusion. After 5 minutes of retrograde perfusion, to allow for temperature equilibration, the left atrium was cannulated in a posterior fashion, and the heart was subsequently perfused in an antegrade (or work-performing) manner against an afterload of $55 \mathrm{~mm}$ Hg. ${ }^{4,8}$

A preload-dependent work-performing experiment was performed in both EC-SOD and CTL hearts according to the following protocol. Preload was gradually increased by raising the height of the antegrade perfusate reservoir, with respect to the left atrium, in $5 \mathrm{~mm} \mathrm{Hg}$ pressure increments from 5 to $25 \mathrm{~mm} \mathrm{Hg}$. At each preload level, left atrial pressure, aortic pressure, and aortic flow data were collected over several cardiac cycles by computer acquisition. After data collection at $25 \mathrm{~mm} \mathrm{Hg}$, the left atrial pressure was slowly lowered to approximately 10 to $12 \mathrm{~mm} \mathrm{Hg}$. Antegrade perfusion was continued for an additional 5 minutes to allow the hearts to recover from any temporary dysfunction that may have potentially been caused by the extreme loading conditions at 20 to $25 \mathrm{~mm}$ $\mathrm{Hg}$.

All hearts were then subjected to 6 minutes of normothermic $\left(38^{\circ} \mathrm{C}\right)$ ischemia, which was achieved by clamping the aortic and left atrial lines. Immediately after ischemia, the hearts were perfused in a retrograde manner for 10 minutes, which was subsequently followed by antegrade perfusion for another 10 minutes at a preload of $10 \mathrm{~mm}$ $\mathrm{Hg}$. The preload-dependent work-performing experiment was again performed to obtain postischemic left atrial pressure, aortic pressure, and aortic flow.
Data acquisition and analysis. All functional and hemodynamic data were digitized on-line at $500 \mathrm{~Hz}$ and filtered by a $50 \mathrm{~Hz}$ low-pass filter, collected, and stored on a microprocessor (PDP 11/23, Digital Equipment Corp., Maynard, Mass.). Raw data were analyzed on a VAX station 3100 computer (Digital Corp, Hitachi, Japan) with software developed in our laboratory, which is previously well described. ${ }^{4}$ Measured on-line parameters included aortic pressure, cardiac output (left ventricular output minus coronary flow), left atrial pressure, and heart rate. Other parameters used to assess preischemic and postischemic myocardial performance were derived from these measured values and included the first derivative of pressure with respect to time ( $\mathrm{dP} / \mathrm{dt})$, stroke volume, and stroke work.

In both CTL and EC-SOD hearts, the average values for each parameter of myocardial performance were calculated from the individual values at preload levels of $5,10,15,20$, and $25 \mathrm{~mm} \mathrm{Hg}$. The percent recovery of each parameter after normothermic ischemia was calculated by dividing the average postischemic value of a specific parameter by its preischemic value.

Myocardial water content. To calculate myocardial water content, every heart was weighed after collection of postischemic preload-dependent functional data, placed in a $120^{\circ} \mathrm{C}$ oven, and dried for 24 hours. The dry weight of each heart was then obtained. Myocardial water content was calculated from the following equation:

Myocardial water content $=$

$$
\frac{(\text { Wet weight }- \text { Dry weight })}{\text { Wet weight }} \times 100 \%
$$

Experimental approval and animal rights. The experimental setup and procedures conformed to the guidelines established by the American Physiological Society and the National Institutes of Health ("Guide for the Care and Use of Laboratory Animals," National Institutes of Health Publication No. 86-23, revised 1985). The experiments were approved by the Duke University Institutional Animal Care and Use Committee (DUIACUC Assigned Registry A485-95-7).

Statistical analysis. Statistical analysis was performed using commercially available software (SigmaStat Version 2.0, Jandel Scientific Software, San Rafael, Calif.). To test for a trend in the various indices of myocardial performance over the entire range of increasing preloads, both before and after normothermic ischemia, a linear multivariate analysis of repetitive measurements was used. Bonferroni's method was used to compensate for the increased risk of a type I error with multiple comparisons. Because this analysis does not indicate which periods differ, follow-up comparison between functional parameters at individual preload levels was performed with paired Student's $t$ tests. Paired Student's $t$ tests were also used to compare the average preischemic data with the average postischemic data within each group. Unpaired Student's $t$ tests were used to compare all data between the EC-SOD and CTL groups. The results are expressed as mean \pm standard error of the mean. A difference was considered statistically significant at $p<0.05$. 


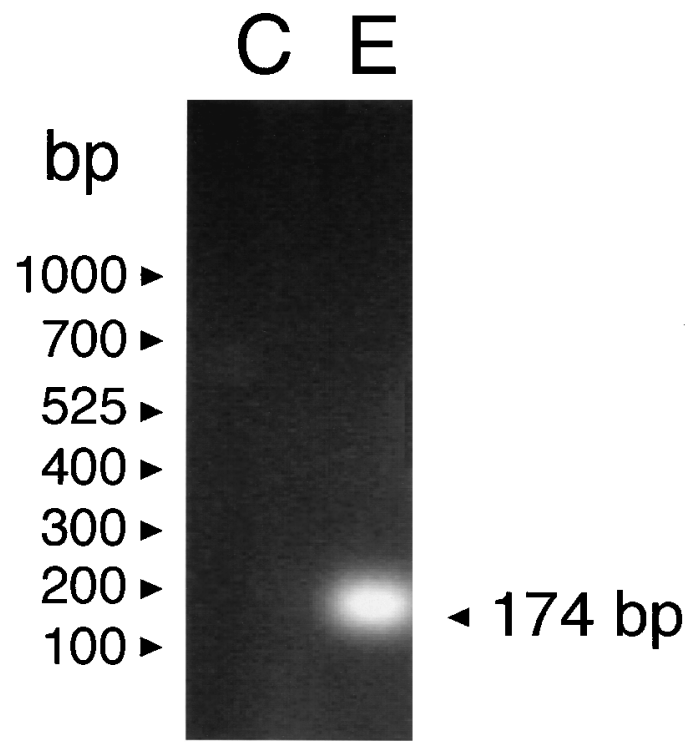

Fig. 2. Reverse-transcriptase polymerase chain reaction analysis. Using this technique, myocardial expression of the 174 base pair $(b p)$ human extracellular superoxide dismutase messenger RNA was found to be present in transgenic mice $(E)$ and absent in control $(C)$ mice.

\section{Results}

Reverse-transcriptase polymerase chain reaction demonstrated that myocardial expression of human SOD messenger RNA was present in the transgenic mice and absent in the control mice (Fig. 2). ECSOD activity was previously found to be 3.5 -fold higher in transgenic hearts compared with control hearts. ${ }^{5}$ Gross examination of both groups revealed no obvious differences in phenotype, breeding, or life span between EC-SOD and CTL mice.

After hypothermic arrest and excision, an average time of $2 \pm 0.5$ minutes was required to cannulate the aorta and connect the heart to the Langendorff apparatus. Mechanical activity resumed almost immediately on initiation of retrograde perfusion with warm Krebs-Henseleit solution. No external pacing was required throughout the experiments.

Myocardial function before and after normothermic ischemia. Before ischemia, increases in cardiac output were observed in association with increases in preload levels for both CTL and EC-SOD mice (Fig. 3). No significant differences were found in the preischemic cardiac outputs of normal and transgenic hearts, which ranged from approximately 3.3 and $6.0 \mathrm{ml} / \mathrm{min}$. After normothermic ischemia, significant decreases in cardiac output were observed in both groups; however, this decrease was signifi-

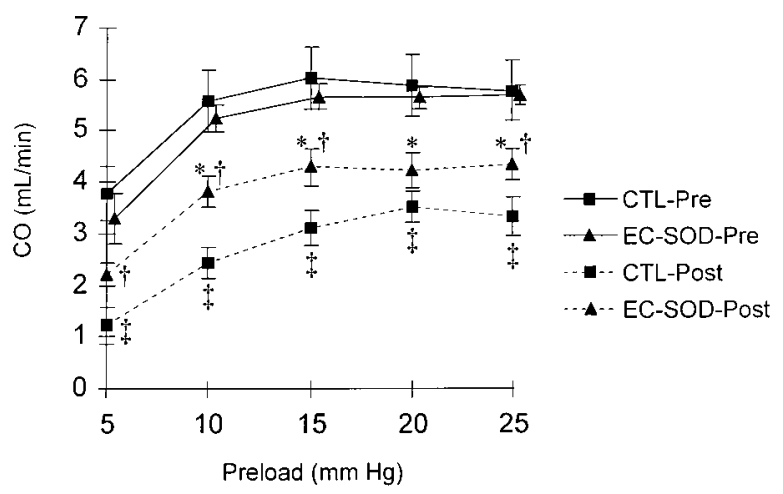

Fig. 3. The preischemic and postischemic cardiac outputs $(\mathrm{CO})$ of both experimental groups are plotted against gradually increasing preload levels at a constant afterload of $55 \mathrm{~mm} \mathrm{Hg}$. Before ischemia, no significant differences were observed in the $\mathrm{CO}$ of CTL mice compared with EC-SOD mice. Significant decreases in CO were observed for both groups after normothermic ischemia; however, this decrease was significantly larger in CTL hearts compared with EC-SOD hearts. *Denotes $p<0.05$ versus EC-SOD-Pre. $\dagger$ Denotes $p<0.05$ versus CTL-Post. $\ddagger D e-$ notes $p<0.01$ versus CTL-Pre.

cantly greater in the CTL group compared with the EC-SOD group (Fig. 3). As a result, the postischemic cardiac output of EC-SOD hearts was significantly greater compared with CTL hearts at preloads of 5, 10, 15, and $25 \mathrm{~mm} \mathrm{Hg}$. Before ischemia, the average cardiac outputs were not significantly different between CTL $(5.40 \pm 0.56 \mathrm{ml} / \mathrm{min})$ and EC-SOD mice ( $5.11 \pm 0.23 \mathrm{ml} / \mathrm{min})$. After ischemia, significant decreases in the average cardiac output were observed in both CTL and EC-SOD hearts; however, the average postischemic cardiac output of EC-SOD hearts $(3.78 \pm 0.28 \mathrm{ml} / \mathrm{min})$ was significantly higher when compared with the average postischemic cardiac output of CTL hearts $(2.73 \pm$ $0.26 \mathrm{ml} / \mathrm{min}, p=0.02$ ).

The average contractility was not significantly different between the two experimental groups before ischemia (Fig. 4). Significant decreases occurred in the average contractility of both CTL and EC-SOD hearts after normothermic ischemia. In addition, the postischemic contractility of EC-SOD mice was higher in comparison with CTL mice; however, this difference was not statistically significant.

The average stroke work values did not differ significantly between CTL hearts and EC-SOD hearts before normothermic ischemia. After ischemia, significant decreases were observed in both 


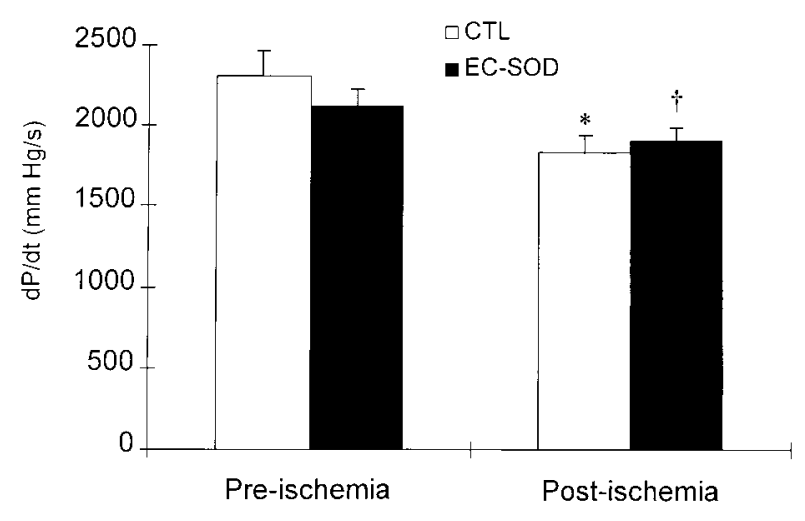

Fig. 4. The differences in the average contractility $(d P / d t)$ between the two experimental groups before and after normothermic ischemia. The average $\mathrm{dP} / \mathrm{dt}$ decreased significantly in both EC-SOD and CTL hearts after normothermic ischemia. *Denotes $p<0.001$ versus $C T L$, Pre-ischemia. †Denotes $p=0.04$ versus EC-SOD, Preischemia.

CTL and EC-SOD mice; however, the average postischemic stroke work of EC-SOD hearts was significantly higher compared with CTL hearts. The differences in the average stroke work between the two experimental groups, before and after ischemia, are displayed in Fig. 5.

On average, the heart rates ranged from 430 to 490 beats/min in both experimental groups. No significant differences were found in the average heart rates between CTL and EC-SOD hearts before ischemia (Fig. 6). Significant decreases were observed in the heart rates of both CTL and ECSOD mice after normothermic ischemia. Although the postischemic heart rates of EC-SOD hearts were increased compared with CTL hearts, these differences were not statistically significant.

Before ischemia, no significant differences in the average stroke volume were observed between CTL mice and EC-SOD mice. After ischemia, a significant decrease in the stroke volume for both CTL and EC-SOD hearts was observed. The postischemic stroke volume of EC-SOD mice, however, was significantly greater compared with the postischemic stroke volume of CTL mice (Fig. 7).

Myocardial water content and percent recovery of postischemic myocardial function. No significant differences were observed in the myocardial water content between CTL $(80.37 \% \pm 0.36 \%)$ and ECSOD hearts $(81.22 \% \pm 0.65 \%)$. The percent recovery of each individual parameter of myocardial performance after normothermic ischemia was sig-

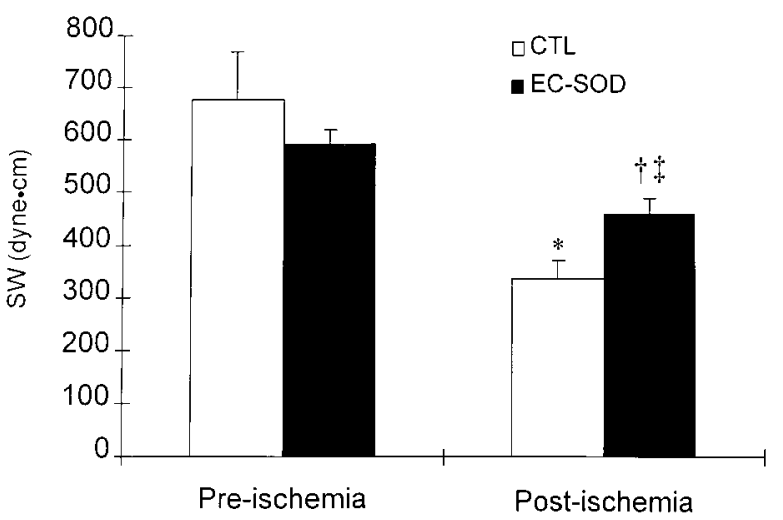

Fig. 5. The differences in the average stroke work $(S W)$ between the two experimental groups before and after normothermic ischemia. The decrease in the average SW of CTL hearts after ischemia was significantly greater compared with the decrease in the average SW of ECSOD hearts. *Denotes $p=0.003$ versus CTL, Pre-ischemia. $\dagger$ Denotes $p=0.005$ versus EC-SOD, Pre-ischemia. $\ddagger$ Denotes $p=0.02$ versus CTL, Post-ischemia.

nificantly larger in the EC-SOD hearts compared with the CTL hearts. These differences in postischemic functional recovery between the two experimental groups are summarized in Table I.

\section{Discussion}

EC-SOD represents one of three distinct types of SOD isoenzymes known to exist in mammalian species. First identified in plasma ${ }^{9,10}$ and later found to exist primarily in the extracellular matrix of tissues ${ }^{11}$ EC-SOD has a relatively low distribution in solid organs compared with the other two SOD isoenzymes, copper-zinc SOD and manganese SOD. It does, however, constitute the predominant form of SOD in vascular tissue, accounting for up to $70 \%$ of total SOD activity, not only in the systemic and pulmonary arteries but also in the coronary circulation. ${ }^{12}$ EC-SOD becomes evenly distributed within the vascular walls after secretion from smooth muscle cells, which make up the principal source of this enzyme. ${ }^{12}$ Although the regulation and importance of both copper-zinc SOD and manganese SOD as antioxidants has been extensively studied and clearly established, the physiologic significance of EC-SOD is, at present, not completely understood. ${ }^{5}$

Numerous investigations have attempted to define the protective role of EC-SOD, particularly with respect to preserving myocardial function in the setting of ischemia and reperfusion injury. ${ }^{13-17}$ Myocardial preservation by EC-SOD after ischemia and 


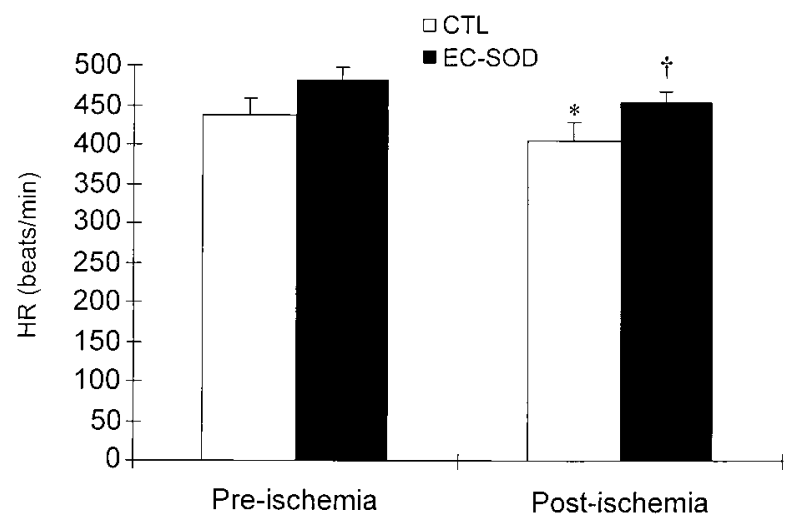

Fig. 6. The differences in the average heart rates (HR) between the two experimental groups before and after normothermic ischemia. The average HR decreased significantly in both EC-SOD and CTL hearts after normothermic ischemia. *Denotes $p=0.03$ versus CTL, Preischemia. †Denotes $p=0.03$ versus EC-SOD, Pre-ischemia.

reperfusion injury, however, has been disputed. Although some investigators have reported a beneficial effect, ${ }^{14,17}$ others have failed to document any protection. ${ }^{15,18}$ This controversy may be related to the method in which the enzyme was administered. In those previous studies, EC-SOD, as well as other antioxidants, was given exogenously at various time intervals around the period of ischemia and reperfusion. These inconsistent approaches among the various investigators would not allow one to draw any firm overall conclusions regarding EC-SOD's protective effect.

Recent advances in molecular biology and in vivo genetic transfer techniques have led to the development of several transgenic animal models with either overexpression or underexpression of very specific genes in the mammalian genome. With respect to myocardial performance, such models have become powerful tools for investigating both normal and altered cardiac physiology and have already led to the greater understanding of adrenergic receptor signaling pathways, ${ }^{19}$ the effects of $\alpha$-skeletal actin overexpression on myocardial contractile function, ${ }^{20}$ and the biochemical triggers responsible for inducing ventricular hypertrophy. ${ }^{21}$ The knowledge gained from such studies has led others to apply this technology further toward developing efficient gene transfer techniques as potentially promising new therapies in the treatment of a number of acquired cardiovascular disorders. ${ }^{22,23}$ Thus overexpression of the EC-SOD in myocardial tissue using a trans-

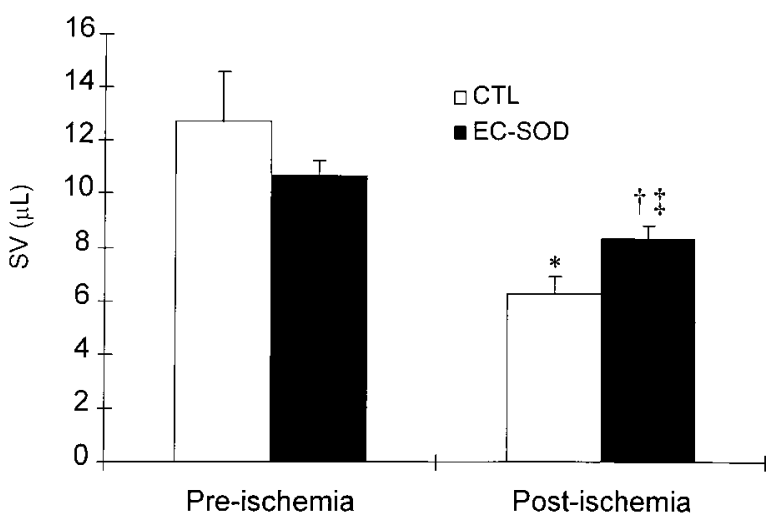

Fig. 7. The differences in the average stroke volume (SV) between the two experimental groups before and after normothermic ischemia. The decrease in the average SV of CTL hearts after ischemia was significantly greater compared with the decrease in the average SV of ECSOD hearts. *Denotes $p=0.002$ versus CTL, Pre-ischemia. $\dagger$ Denotes $p=0.006$ versus EC-SOD, Pre-ischemia. $\ddagger$ Denotes $p=0.03$ versus CTL, Post-ischemia.

genic model appears to offer a unique strategy for first investigating the myocardial protective effects of EC-SOD after ischemia and reperfusion injury.

The isolated work-performing heart (Langendorff) preparation represents a highly sensitive and previously validated model of evaluating both preischemic and postischemic cardiac function in isolated murine hearts. ${ }^{4}$ In contrast to other Langendorff systems, the present apparatus uses both micromanometers and an ultrasonic flow probe in combination with computerized data acquisition. Such high-fidelity instruments allow for the recording of heart rates of up to 1500 to 1800 beats/min and for analysis of very sensitive parameters of myocardial performance such as stroke work. ${ }^{4}$ In the only other well-described isolated work-performing heart preparation used for evaluating myocardial performance in murine hearts, fluid-filled pressure catheters were used to obtain intracavitary pressures, contractility, and heart rates. ${ }^{8}$ Because the physiologic heart rates observed in this study ranged from 400 beats/min to more than 500 beats/ min, application of such catheters in this setting might lead to dampening and distortion of the pressure waveforms, as well as a decreased frequency response at the time of data acquisition.

A potential criticism of this model is that KrebsHenseleit solution, instead of blood, was used for both retrograde and antegrade myocardial perfusion. A blood-perfused, isolated work-performing 
Table I. Percent recovery (\% Rec) of preload-dependent myocardial function after normothermic ischemia

\begin{tabular}{llllll}
\hline & & & $d P / d t$ & & \\
& $C O(\% \mathrm{Rec})$ & $S W(\% \mathrm{Rec})$ & $(\% \mathrm{Rec})$ & $\mathrm{HR}(\% \mathrm{Rec})$ & $S V(\% \mathrm{Rec})$ \\
\hline CTL & $49.66(2.40)$ & $54.78(2.95)$ & $78.15(1.86)$ & $87.04(1.97)$ & $54.15(2.80)$ \\
EC-SOD & $73.25^{*}(3.27)$ & $77.41^{*}(3.96)$ & $90.15 \dagger(3.61)$ & $94.33 \div(2.03)$ & $77.90^{*}(3.90)$ \\
\hline
\end{tabular}

$C O$, Cardiac output; $S W$, stroke work; $d P / d t$, average contractility; $H R$, heart rate; $S V$, stroke volume; $C T L$, control hearts; $E C$-SOD, extracellular superoxide dismutase overexpressed hearts.

${ }^{*} p<0.001$ versus CTL.

$\dagger p=0.01$ versus $\mathrm{CTL}$.

$\ddagger p=0.03$ versus CTL.

SEM in parentheses.

murine heart preparation would certainly be more physiologic than use of any crystalloid solution. The glutathione in red blood cells might also substantially limit the ischemia and reperfusion-induced burst of oxygen-derived free radicals and subsequent cardiac injury. Although the present design can potentially exaggerate myocardial damage after ischemia and subsequent reperfusion, such conditions were identical for both experimental groups. In addition, it must be pointed out that set up of the Langendorff system in this report before actual experimentation requires at least 1500 to $1800 \mathrm{ml}$ of priming solution. Thus an inordinate number of mice, given the small body size and plasma volume, would be needed to obtain enough blood for one set of experiments. Even if dilute blood were used as the perfusate, such requirements seem somewhat impractical.

Before ischemia, no significant differences were observed in any parameter of cardiac function between the two experimental groups. Significant decreases in myocardial performance were observed in both transgenic and nontransgenic hearts after global normothermic ischemia; however, these decreases were significantly greater in the nontransgenic hearts compared with the transgenic hearts. As a result, the postischemic recovery of each measured parameter of cardiac function was significant greater in mice with myocardial overexpression of EC-SOD compared with littermate CTL mice.

Thus transgenic overexpression of EC-SOD in isolated murine hearts does not appear to affect baseline myocardial performance compared with normal hearts. The significantly higher percent recovery of postischemic function, observed in hearts overexpressing EC-SOD, suggests that this specific antioxidant may have an important role in preserving myocardial function after ischemia and reperfu- sion injury. Additional insight could be gained if myocardial recovery were also assessed in mice with greater than 3.5-fold overexpression of EC-SOD; however, creation of such an animal has not been previously described.

The decision to use a 6-minute period of global normothermic ischemia was made after a series of preliminary studies in which the ischemic interval was varied from 4 to 7 minutes in normal murine hearts. A dose-response curve was subsequently generated, demonstrating that 4 to 5 minutes of ischemia resulted in a $25 \%$ to $30 \%$ decrease in cardiac output, whereas 6 minutes led to a reduction in cardiac output of $45 \%$ to $50 \%$. In addition, no appreciable functional recovery was observed in control hearts after 7 minutes of global ischemia. Because it was initially unclear as to what effect myocardial EC-SOD overexpression would have on postischemic recovery of cardiac performance, 6 minutes was believed to be the most appropriate period of normothermic ischemia for this study. One can argue that such a brief period of ischemia has little clinical relevance to the clinical setting. It should be pointed out, though, that the purpose of these preliminary studies was only to determine a period of ischemia that would lead to a significant reduction of cardiac performance in murine hearts to adequately assess the myocardial protective effects of EC-SOD.

Nonetheless, it was somewhat surprising that the control hearts in this study were so sensitive to ischemia, requiring only 6 minutes to effect a $50 \%$ reduction in preload-dependent myocardial performance. This finding, however, is consistent with another investigation involving normal wild-type murine hearts, in which a $40 \%$ recovery of developed pressure was observed after 6 minutes of global ischemia. ${ }^{24}$ On the other hand, previous studies using rat hearts have shown that at least 15 
to 30 minutes of normothermic ischemia is required to impair myocardial function by $50 \% .^{17,22,25,26} \mathrm{~A}$ potential explanation for this apparent discrepancy may lie in the differences in baseline heart rates between these two species.

In an earlier investigation comparing adenosine triphosphate (ATP) depletion and mitochondrial functional loss during ischemia between slow (dog) and fast (rat) heart-rate hearts, Rouslin and associates $^{27,28}$ demonstrated that slow heart-rate hearts use a more diverse set of mechanisms for conserving ATP during ischemia than fast heart-rate hearts, which includes a mitochondrial $F_{1}$-ATPase inhibitor protein. Fast heart-rate hearts generally rely on a single mechanism for maintaining high-energy phosphate during ischemia, namely, anaerobic glycolysis. $^{27}$ These differences suggest that the greater diversity of mechanisms for conserving ATP used by slow heart-rate hearts may be a more effective strategy for maintaining cellular ATP stores and thereby preserving cellular function during ischemia. $^{27}$

Admittedly, mice, along with rats, are fast heartrate animals. ${ }^{28}$ Nevertheless, a marked difference exists in the heart rates between these two species. Previous studies demonstrate that baseline rat heart rates range from approximately 230 to 270 beats/ min, ${ }^{17}$ which are much slower than the murine heart rates of 430 to 490 beats/min observed in this report. Thus the observed sensitivity of murine hearts to brief periods of ischemia compared with rat hearts could be due to a much faster depletion of highenergy phosphates resulting from higher metabolic demand. Quantitation of myocardial ATP levels in murine hearts during progressively longer periods of ischemia from 4 to 7 minutes may provide a biochemical explanation for the rapid decline in function, but these comparisons require further investigation.

The present findings might initially appear to contradict those in the original description of the isolated work-performing murine heart preparation, in which a 7-minute period of ischemia in normal murine hearts resulted in a $22 \%$ to $24 \%$ reduction in cardiac output and stroke work and no significant changes in either the heart rates or stroke volumes. ${ }^{4}$ It is important to keep in mind, however, that all hearts in this report were subjected to periods of ischemia that occurred under normothermic conditions at $38^{\circ} \mathrm{C}$. In the previous study, hearts were exposed to warm, and slightly hypothermic, temperatures of $32^{\circ} \mathrm{C}$ during the 7 -minute ischemic inter- val. Thus the degree of injury was not precisely identical for the two studies, and any differences in the recovery of postischemic myocardial performance would certainly be expected.

It would be interesting to determine whether periods of hypothermic ischemia would have effects on EC-SOD overexpressed hearts similar to normothermic ischemia because this scenario is quite analogous to the clinical setting in which cardioplegia is often used to induce a period of hypothermic arrest at the time of cardiac surgery. Unfortunately, one cannot predict, from the data presented, whether these transgenic mice would respond to hypothermia in a comparable manner to the way they recovered from normothermic ischemia. Any conclusions regarding such a response necessitate additional investigation.

The significant protective effect of EC-SOD observed at the physiologic level in this report warrants further investigation into the potential biochemical mechanisms behind such protection. A compelling mechanistic thread could be constructed if significantly lower levels of oxygen-derived free radicals and decreased oxidant-mediated injury (through lipid peroxidation and sulfhydryl group oxidation) were documented in hearts of transgenic mice after ischemia and reperfusion injury. Such mechanisms, however, were not the subject of these experiments; the goal of this report was to first establish a physiologic protective effect. It would be prudent to next proceed into examining the molecular mechanisms behind EC-SOD's myocardial protective effects. Although purely speculative, this information could eventually be applied clinically for administering EC-SOD, through the use of gene transfer techniques, as a novel therapy in treating myocardial ischemia and reperfusion injury.

In summary, transgenic overexpression of human EC-SOD in isolated murine hearts does not appear to affect baseline myocardial performance compared with normal hearts. After global normothermic ischemia and subsequent reperfusion, significant decreases in cardiac function were observed in both transgenic and nontransgenic hearts. A significantly higher percentage of functional recovery, however, was observed in EC-SOD overexpressed hearts. The experimental model should now be applied in assessing potential biochemical mechanisms, occurring by way of the antioxidant pathways of EC-SOD, which may play an important role in the preservation of myocardial function after ischemia and reperfusion injury. 


\section{REFERENCES}

1. Werns SW, Luccesi BR. Myocardial ischemia and reperfusion: the role of oxygen free radicals in tissue injury. Cardiovasc Drugs Ther 1989;2:761-9.

2. Folz RJ, Crapo JD. Pulmonary oxygen toxicity. Curr Pulmonology 1994;15:113-26.

3. McCord JM, Fridovich I. Superoxide dismutase: an enzymatic function for erythrocuprein (hemocuprein). J Biol Chem 1969;244:6049-55.

4. Bittner HB, Chen EP, Peterseim DP, Van Trigt P. A work-performing heart preparation for myocardial performance analysis in murine hearts. J Surg Res 1996;64:57-62.

5. Oury TD, Ho YS, Piantadosi CA, Crapo JD. Extracellular superoxide dismutase, nitric oxide, and central nervous system $\mathrm{O}_{2}$ toxicity. Proc Natl Acad Sci U S A 1992;89:9715-9.

6. Sambrook J, Fritsch EF, Maniatis T. Molecular cloning: a laboratory manual. 2nd ed. Plainview (NY): Cold Spring Harbor Laboratory Press, 1989. p. 5.52-5.55.

7. Crapo, JD, McCord JM, Fridovich I. Preparation and assay of superoxide dismutases. Methods Enzymol 1978;53:382-93.

8. Grupp IL, Subramaniam A, Hewett TE, Robbins J, Grupp G. Comparison of normal, hypodynamic, and hyperdynamic mouse hearts using isolated work-performing heart preparations. Am J Physiol 1993;265:H1401-10.

9. Marklund SL. Human copper-containing superoxide dismutase of high molecular weight. Proc Natl Acad Sci U S A 1982;79:7634-8.

10. Marklund SL, Holme E, Hellner L. Superoxide dismutase in extracellular fluids. Clin Chim Acta 1982;126:41-51.

11. Marklund SL. Extracellular superoxide dismutase and other superoxide dismutase isoenzymes in tissues from nine mammalian species. Biochem J 1984;222:649-55.

12. Strålin P, Karlsson K, Johansson BO, Marklund SL. The interstitium of the human arterial wall contains very large amounts of extracellular superoxide dismutase. Arterioscler Thromb Vasc Biol 1995;15:2032-6.

13. Omar BA, McCord JM. Interstitial equilibrium of superoxide dismutase correlates with its protective effect in the isolated rabbit heart. J Mol Cell Cardiol 1991;23:149-59.

14. Hatori N, Sjöquist P, Marklund SL, Ryden L. Effects of recombinant human extracellular superoxide dismutase type $\mathrm{C}$ on myocardial infarct size in pigs. Free Radic Biol Med 1992;13:221-30.

15. Menasche P, Grousett C, Gauduel Y, Piwnica A. A comparative study of free radical scavengers in cardioplegic solutions. J Thorac Cardiovasc Surg 1986;92:264-71.

16. Sjöquist P, Marklund SL. Endothelium bound extracellular superoxide dismutase type $\mathrm{C}$ reduces damage in reperfused ischaemic rat hearts. Cardiovasc Res 1992;26:347-50.

17. Hatori N, Sjöquist P, Marklund SL, Pehrsson SK, Ryden L. Effects of recombinant human extracellular superoxide dismutase type $\mathrm{C}$ on myocardial reperfusion injury in isolated cold-arrested rat hearts. Free Radic Biol Med 1992;13:13742.

18. Myers CL, Weiss SJ, Kirsh MM, Shlafer M. Involvement of hydrogen peroxide and hydroxyl radical in the "oxygen paradox": reduction of creatine kinase release by catalase, allopurinol or deferoxamine, but not by superoxide dismutase. J Mol Cell Cardiol 1985;17:675-84.

19. Koch WJ, Milano CA, Lefkowitz RJ. Transgenic manipulation of myocardial $\mathrm{G}$ protein-coupled receptors and receptor kinases. Circ Res 1996;78:511-6.
20. Hewett TE, Grupp IL, Grupp G, Robbins J. $\alpha$-Skeletal actin is associated with increased contractility in the mouse heart. Circ Res 1994;74:740-6.

21. Milano CA, Dolber PC, Rockman HA, et al. Myocardial expression of a constitutively active $\alpha_{1 \mathrm{~B}}$-adrenergic receptor in transgenic mice induced cardiac hypertrophy. Proc Natl Acad Sci U S A 1994;91:10109-13.

22. Sawa Y, Kadoba K, Suzuki K, et al. Efficient gene transfer technique into the whole heart through the coronary artery with hemagglutinating virus of Japan liposome. J Thorac Cardiovasc Surg 1997;113:512-9.

23. Rowland RT, Cleveland JC, Meng X, Harken AH, Brown JM. Potential gene therapy strategies in the treatment of cardiovascular disease. Ann Thorac Surg 1995;60:721-8.

24. Radford NB, Fina M, Benjamin IJ, et al. Cardioprotective effects of 70-kDa heat shock protein in transgenic mice. Proc Natl Acad Sci U S A 1996;93:2339-42.

25. Pasque MK, Spray TL, Pellom GL, et al. Ribose-enhanced myocardial recovery following ischemia in the isolated working rat heart. J Thorac Cardiovasc Surg 1982;83:390-8.

26. Schaefer S, Ramasamy R. Glycogen utilization and ischemic injury in the isolated rat heart. Cardiovasc Res 1997;35:90-8.

27. Rouslin W, Broge CW, Grupp IL. ATP depletion and mitochondrial functional loss during ischemia in slow and fast heart-rate hearts. Am J Physiol 1990;259:H1759-66.

28. Rouslin W. The mitochondrial adenosine 5 '-triphosphatase in slow and fast heart rate hearts. Am J Physiol 1987;252: H622-7.

\section{Discussion}

Dr. Pedro J. del Nido (Boston, Mass.). First of all, I congratulate you for using a model that more directly addresses the question of the role of SOD in ischemiareperfusion, and I would encourage the use of this kind of model. The problem, however, is that compensatory mechanisms always occur in these transgenic animals.

My first question relates to the fact that it is known that these animals live longer and, therefore, age more slowly. You have weight-matched animals. Are they age-matched as well? In other words, are you comparing younger animals to older animals simply because you use weight as your control?

And the second question is, what about other antioxidant enzymes? If you up-regulated SOD, do you also have up-regulation of catalase or glutathione peroxidase, which is what one would expect in this model?

Dr. Chen. Thank you, Dr. del Nido. We certainly agree with you that transgenic overexpression represents a very unique and direct model as well. Several of the other reports that have been published regarding the potential protective effects of EC-SOD have involved exogenous administration around the ischemic period. We believe the different methods used by different investigators would not allow for any firm overall conclusions.

These mice were actually age-matched. And by gross examination, no difference was observed in the phenotype or life span between EC-SOD and CTL mice.

As far as other compensatory mechanisms, that is certainly something we had considered as well.

Regarding the other two types of SOD isoenzymes, 
copper-zinc SOD and manganese SOD, no difference in expression was observed between the transgenic and nontransgenic mice. We had planned to assess catalase and glutathione activity between the two groups; however, that has not been done at this time.

Dr. John E. Mayer, Jr. (Boston, Mass.). What if you turn the experiment around the other way, have you made knockout mice for this enzyme?

Dr. Chen. That was a very good point that we recognized as well along with our collaborators. Those mice have been created; however, they have not been studied on this apparatus.

Dr. Yoshifumi Naka (New York, N.Y.). Have you looked at the biochemical parameters that could suggest reduction of superoxide amount?

Dr. Chen. We have not. In this study these hearts were weighed and then oven-dried to obtain dry weights. We then calculated myocardial water content, which was not significantly different between the two groups. We had planned another set of experiments in which we would subject additional hearts to these same experimental conditions and assess biochemical levels of oxidant-free radicals and SOD activity.

Dr. Naka. Have you tried similar experiments using the transgenic mice with EC-SOD-like protein that is encoded similar to EC-SOD but different from EC-SOD? That is why this protein has the same molecular weight and a similar one-dimension configuration but no or little affinity to SOD. My point is that you demonstrated starting and end points of the black box, superoxide dismutation by EC-SOD, by showing improved physiologic parameters, but you have shown no direct evidence of this phenomenon. A theoretical concern is that overexpressed and increase protein may cause improved function.

Dr. Chen. We have not examined other types of transgenic mice with proteins or enzymes that are similar in structure to EC-SOD but do not contain the activity. 